\title{
Colorectal surgery for a centenarian patient
}

\author{
Georgios C. Sotiropoulos • Nikolaos Machairas • Peter Tsaparas • \\ Paraskevas Stamopoulos • Gregory Kouraklis
}

Accepted: 22 February 2015 / Published online: 5 March 2015

(C) Springer-Verlag Berlin Heidelberg 2015

Dear editor:

Recent developments in surgical techniques, anesthesia, and perioperative care have made surgical therapy approachable for the very aged patients. Surgical series on septuagenarians and octogenarians have already been published and patient age is not any more considered as a contraindication per se even for major operations. However, although the indication for surgery in advanced age is personalized, no upper limit has been determined yet. We report herein on a successful urgent operation in a 100 -year-old patient.

A centenarian was admitted to the emergency room of our hospital, suffering from meteorism with mild abdominal pain. He was in an acceptable general condition, well orientated, and not bedridden and was able to perform some basic activities in his daily life, according to the testimony of his nephew, who was living with him. Abdominal X-ray showed a massive large intestine ileus. Abdominal sonography documented the colonic ileus and the presence of ascetic fluid. A colonoscopic decom- pression was initiated, which failed due to a mechanical obstruction in the upper rectum. As the clinical condition of the patient was deteriorated, decision for operative management was taken. The patient underwent a mini-upper abdominal laparotomy and a placement of a double-barreled transverse colostomy under general anesthesia. The patient tolerated the operation well and experienced an uneventful postoperative course. He was discharged on postoperative day 8 with dietary instructions. Further investigations and eventually oncologic treatment were denied from his family. The patient gradually restored his preoperative general condition and is alive 18 months post-surgery.

This case underlines on the one hand the feasibility of performing colorectal surgery even in an emergency situation in a centenarian and on the other hand the slow growth of the presumed rectal tumor in this age. To the best of our knowledge, this is the first report of a successful colon operation, even in the form of colostomy, in a centenarian, with a postoperative follow-up of more than 1 year.

G. C. Sotiropoulos $(\bowtie) \cdot$ N. Machairas $\cdot$ P. Tsaparas •

P. Stamopoulos · G. Kouraklis

2nd Department of Propedeutic Surgery, University of Athens

Medical School, Laikon Hospital, 17 Agiou Thoma Street,

Athens 11527, Greece

e-mail: georgios.sotiropoulos@uni-due.de 